D) Check for updates

Cite this: Polym. Chem., 2020, 11, 5088

Received 11th May 2020,

Accepted 24th June 2020

DOI: $10.1039 / \mathrm{d} 0$ py00688b

rsc.li/polymers

\title{
Monitoring the structure-reactivity relationship in epoxidized perilla and safflower oil thermosetting resins $\uparrow$
}

\author{
Thi-Nguyet Tran, ${ }^{a}$ Chiara Di Mauro, ${ }^{a}$ Alain Graillot (D) ${ }^{\mathrm{b}}$ and Alice Mija (D) *a
}

\begin{abstract}
For the first time, the effect of reactant structure, stoichiometry and heating rate on the reactivity of epoxidized perilla oil (EPLO) and epoxidized safflower oil (ESFO) with dicarboxylic acids (DCAs) was studied using in situ FT-IR. The epoxy content in the monomer structure was found to affect the copolymerization system's reactivity, with epoxidized linseed oil (ELO) considered as a reference. In this study we discuss also the influence of the DCA structure on the copolymerization reactivity. Two aromatic diacids, dithiodibenzoic acid (DTBA) and diphenic acid (DPA), were studied and compared in the copolymerization of the $3 \mathrm{EVOs}$, in the presence of imidazole (IM) initiator. The kinetics of these reactions were followed by in situ FT-IR. The corresponding activation energies were calculated via different kinetic models. These data highlight the higher reactivity of the EPLO monomer and the DTBA hardener.
\end{abstract}

\section{Introduction}

Significant emphasis on serious environmental pollution, restriction of petroleum oil resources, greenhouse gas (GHG) emission, and waste accumulation prompt many scientists to focus on the sustainable development of the chemical industry. ${ }^{1-4}$ Vegetable oils (VOs) as raw materials are considered to be some of the most promising bioresources because of their availability, advantageous costs, and especially their wide variety of chemical transformations. ${ }^{2,5-10}$ One common strategy to obtain polymeric materials from VOs is the polymerization of the $\mathrm{C}=\mathrm{C}$ double bonds on their backbone. Another approach is based on their functionalization. For example VOs can be functionalized with epoxy groups in a sustainable way. These methods involve the action of hydrogen peroxide and acetic or formic acid, generating the corresponding epoxidized vegetable oils (EVOs), nontoxic products with higher chemical reactivity compared to VOs. The presence of reactive functional groups together with the possibility of producing them on a large scale make EVOs extremely attractive as green monomers for the bio-based thermosetting resins market. ${ }^{11-14,15}$ In general, EVOs are mainly composed of trigly-

\footnotetext{
${ }^{a}$ Université Côte d'Azur, Institut de Chimie de Nice, UMR 7272 - CNRS, 28 Avenue Valrose, 06108 Nice Cedex 2, France. E-mail: Alice.MIJA@univ-cotedazur.fr

${ }^{b}$ SPECIFIC POLYMERS, 150 Avenue des Cocardières, Zac Via Domitia, 34160 Castries, France

$\dagger$ Electronic supplementary information (ESI) available: FT-IR spectral assignment tables for raw materials; table of EVO epoxy content and molar mass. See DOI: $10.1039 /$ d0py00688b
}

ceride molecules, which are constituted of three fatty acids bound by a glycerol center through ester linkages. ${ }^{16,17}$ Although their (macro)molecular structures are similar, different epoxidized vegetable oils have different reactivity from each other. This is related to the chain length, and the number and positions of epoxy groups. The curing process of EVOs has been investigated in numerous studies, ${ }^{1,2,8,18-22}$ however, to the best of our knowledge, no one has performed a comparison of the structure-reactivity relationship of different EVOs.

The choice of an appropriate curing agent is very important to achieve the designed thermomechanical properties of the cured epoxy resins. ${ }^{23-25}$ Among several classes of curing agent such as amines, anhydrides, thiols, polyols, etc., carboxylic acids are specifically selected in this study due to their relatively low price, the widespread availability of the raw materials and the good flexibility and weatherability properties of the resulting materials. ${ }^{26}$ Recently, a new generation of polymer networks has been developed, called "vitrimers", in which reversible/dynamic chemistries are introduced. These materials offer a possible solution for reprocessing or recycling thermoset materials, thus reducing the impact on the environment and improving the materials' end of life as well as being a circular economy. The most common dynamic or reversible chemistries used in the creation of vitrimer materials are carboxylate transesterifications, ${ }^{27-29}$ the transalkylation of triazolium salts, ${ }^{30}$ olefin metathesis, ${ }^{31}$ disulfide exchange, ${ }^{32,33}$ etc.

In many reported studies, materials reprocessing usually takes place with the help of an external stimulus such as UV irradiation $^{34,35}$ or heating at high temperature, ${ }^{36}$ or in the pres- 
ence of a catalyst. ${ }^{27,37}$ Distinguished from other materials, sulfur-containing moieties (S-S) offer unique opportunities in which exchange can be activated at moderate temperature without any catalyst or initiator. ${ }^{32,38-40}$ Inspired by the strategy of importing exchange bonds from the hardener structure, we used disulfide-containing acids as crosslinkers to design biobased thermosets with dual dynamic exchange: through (i) carboxylate transesterification, and (ii) disulfide metathesis.

As the properties of polymers depend strongly on their structures, in the case of epoxy thermosets they depend on the structure of both the epoxy monomer and the curing agent. For example, in the case of EVO-based thermosets, ${ }^{41-44}$ a low mechanical strength could result from systems with high content of flexible backbones and/or low crosslink density. In contrast, the use of appropriate curing agents could reinforce significantly the strength and modulus of EVO-based thermosets. Therefore, the most important key parameter in materials development is the structure-reactivity-properties relationship. It can help to predict and therefore to design the properties and performances of the final products formed through a given manufacturing process. In this context, this work reports for the first time the comparison of 3 EVO crosslinking reactions with aromatic diacids, and the structurereactivity relationships and kinetics were studied through FT-IR.

To monitor the cure kinetics of thermosetting resin, Fourier Transform Infrared (FT-IR) spectroscopy and Differential Scanning Calorimetry (DSC) are two common techniques. DSC experiments can be performed to obtain kinetic parameters, which make it possible to simulate the curing. However, DSC only gives information about the overall process and not about the elemental reactions that occur during curing. Therefore, FT-IR spectroscopy is specifically selected as an interesting alternative to monitor the curing kinetics. Indeed, mid-infrared spectroscopy is the most suitable technique for following epoxy-hardener crosslinking reactions. It should be noted that many FT-IR spectral frequencies change in a significant way during this reaction, correlated to the nature and number of reacting groups in the crosslinking mixture. These changes can be monitored. Both qualitative and quantitative information can be obtained using this technique which provides a direct measurement of the evolution in concentration over time and temperature of any chemical group involved in the reaction. Furthermore, this method allows us to process the data and therefore to compute the kinetic parameters of the investigated reaction, i.e. conversion degree $(\alpha)$, reaction rate $(\mathrm{d} \alpha / \mathrm{d} t)$, and activation energy $\left(E_{\mathrm{a}}\right)$.

\section{Results and discussion}

Previously, we demonstrated that the use of imidazole as initiator provides efficient epoxy/acid crosslinking, generating thermosets with tunable thermomechanical properties. ${ }^{45}$ In the present study, we selected 3 different EVO monomers with three levels of epoxide content varying from 3.7 to 8.0 equiva- lents per mole. The curing of these 3 EVOs with 2 dicarboxylic acids (DCAs), namely 2,2'-dithiodibenzoic acid (DTBA) and diphenic acid (DPA), was investigated by in situ FT-IR analysis. The effect of epoxy monomer and crosslinker on the curing kinetics is studied in this work.

\section{General characteristics of the FT-IR spectra}

Epoxidized vegetable oils (EVOs). From the FT-IR spectra illustrated in Fig. 1 we can firstly observe that the 3 EVOs, i.e. EPLO with 8 eq. epoxide per mole, ELO with 5.5 eq. epoxide per mole and ESFO with 3.7 eq. epoxide per mol, have very similar absorptions, since their structures are also very similar. The principal FT-IR absorption peaks are: $-\mathrm{CH}_{2}$ asymmetric stretching at $2947 \mathrm{~cm}^{-1} ;-\mathrm{CH}_{2}$ symmetric stretching at $2854 \mathrm{~cm}^{-1}$; and $-\mathrm{C}=\mathrm{O}$ stretching of the ester triglycerides at $1740 \mathrm{~cm}^{-1}$. The asymmetric $\mathrm{C}-\mathrm{O}$ stretching band of the ester groups appears at $1150 \mathrm{~cm}^{-1}$ and the $\mathrm{C}-\mathrm{O}$ ether stretching bands for the $\alpha$-positions of the ester groups are located at 1095 and $1115 \mathrm{~cm}^{-1}$.4,46,47 Two specific absorptions of the oxirane ring are observed in the range between $4000 \mathrm{~cm}^{-1}$ and $400 \mathrm{~cm}^{-1}$. The first one, at about $823-847 \mathrm{~cm}^{-1}$, is attributed to the $\mathrm{C}-\mathrm{O}$ deformation of the oxirane group. ${ }^{46,48}$ The second band is located at $\sim 3050 \mathrm{~cm}^{-1}$ and is attributed to the $\mathrm{C}-\mathrm{H}$ stretching of the methylene group of the epoxy ring. Due to its low intensity and very close vicinity to the strong $\mathrm{O}-\mathrm{H}$ absorption, it is preferable to use the first band, located at about $823 \mathrm{~cm}^{-1}$, to quantify the conversion degree of epoxy groups.

Dicarboxylic acid hardeners. The common peak assignments of the two selected DCA hardeners are summarized in Table S1.† Similar to all carboxylic acids, the $\mathrm{O}-\mathrm{H}$ stretching vibration band is very broad in the region of $3300-2300 \mathrm{~cm}^{-1}$ with a maximum at $2813 \mathrm{~cm}^{-1}$ (Fig. 2). This band is overlapped with the stretching vibration region of the aromatic $\mathrm{C}-\mathrm{H}$ groups. We observe also other common characteristic peaks including: $\mathrm{C}=\mathrm{O}$ stretching at about $1674 \mathrm{~cm}^{-1} ; \mathrm{C}-\mathrm{O}$ stretching at 1320-1000 $\mathrm{cm}^{-1}$ and $\mathrm{O}-\mathrm{H}$ bending at $\sim 1450 \mathrm{~cm}^{-1}$ and $\sim 900 \mathrm{~cm}^{-1} \cdot{ }^{12,49,50}$ The characteristic peaks in the aromatic region are observed at $\sim 1590$ and $\sim 740 \mathrm{~cm}^{-1}$ for the two orthosubstituted benzene rings.

\section{EPLO/DTBA/IM system}

A FT-IR comparison of the starting materials with the reactive EPLO/DTBA/IM mixture and the thermoset is presented in

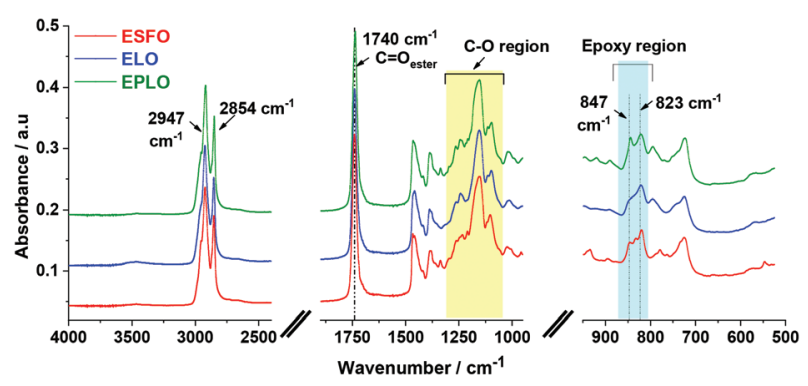

Fig. 1 FT-IR spectra of the three EVO monomers, namely EPLO, ELO, and ESFO. 

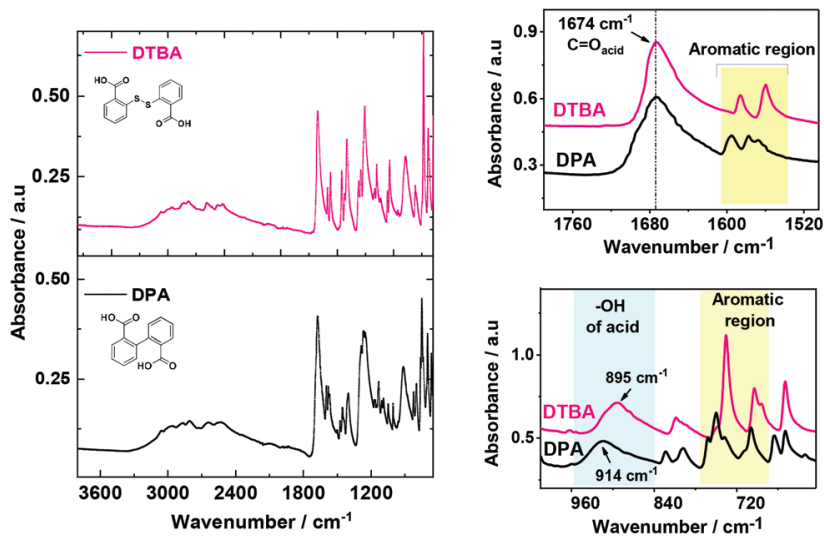

Fig. 2 FT-IR spectra of the two hardeners: 2,2'-dithiodibenzoic acid (DTBA) and diphenic acid (DPA).

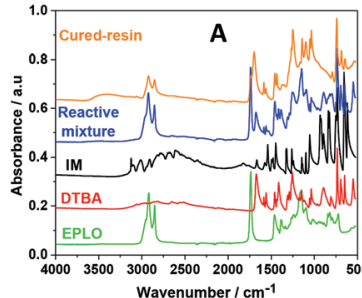

Wavenumber $/ \mathrm{cm}^{-1}$

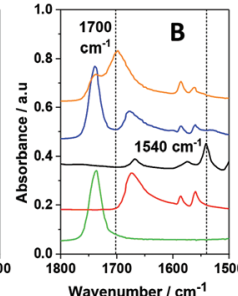

Wavenumber $/ \mathrm{cm}^{-1}$

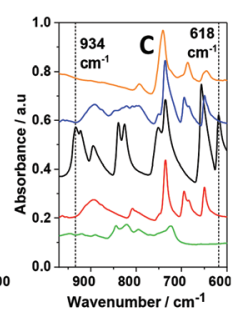

Fig. 3 FT-IR spectra of the starting materials, namely EPLO, DTBA, and $I M$, in comparison with the reactive mixture at the initial time and the corresponding cured resin: (A) whole spectrum from 4000 to $500 \mathrm{~cm}^{-1}$; (B) close-up of the region from 1800 to $1500 \mathrm{~cm}^{-1}$; (C) close-up from 950 to $600 \mathrm{~cm}^{-1}$

Fig. 3. Table S2 $\uparrow$ summarizes the corresponding assignments of the IM absorption bands. Two bands for IM at about 823-847 $\mathrm{cm}^{-1}$, corresponding to $\mathrm{N}-\mathrm{H}$ out-of-plane bending and in-plane ring deformation, are located in the same region as one of the bands of the EPLO oxirane ring. However, we can remark that the two strong absorption peaks of IM at 934 and $618 \mathrm{~cm}^{-1}$ together with the peak at $1540 \mathrm{~cm}^{-1}$ are missing in the FT-IR spectrum of the reactive mixture, at the initial time. Considering that only $1 \mathrm{wt} \%$ of IM is included in the reactive mixture, the lack of peaks indicates that the IM signals are negligible compared to those of EPLO and DTBA.

To evaluate the interactions of each component during the imidazole catalyzed epoxy-acid crosslinking, in situ FT-IR measurements were performed on the mixtures EPLO/DTBA/ IM, EPLO/IM and DTBA/IM using the same heating rate from $25{ }^{\circ} \mathrm{C}$ to $180{ }^{\circ} \mathrm{C}$. The corresponding spectra are displayed in Fig. 4.

Evaluation of the peak area for the epoxy groups at about 823-847 $\mathrm{cm}^{-1}$ allows us to follow the progress of the ring opening reaction. The extent of the crosslinking reaction was quantified by the peak area for the epoxy group absorption at about $823 \mathrm{~cm}^{-1}$ in reference to the internal standard. Two methods were applied to calculate the epoxy conversion, using eqn (5) as described in the Experimental section of the ESI. $\dagger$

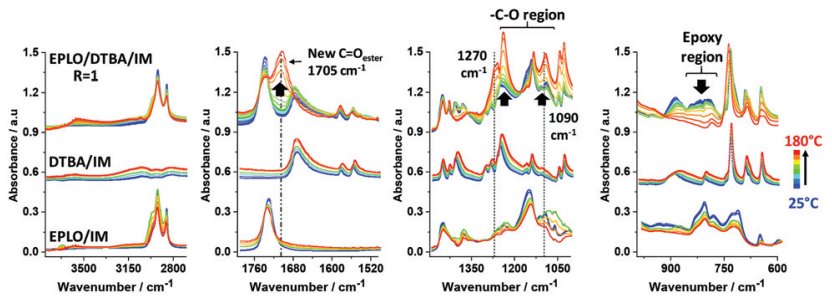

Fig. 4 Evolution of FT-IR spectra during heating from $25^{\circ} \mathrm{C}$ to $180^{\circ} \mathrm{C}$ at $10^{\circ} \mathrm{C} \mathrm{min}^{-1}$, comparing the 3 mixtures: (1): EPLO/DTBA/IM; (2) DTBA/ IM; (3): EPLO/IM.
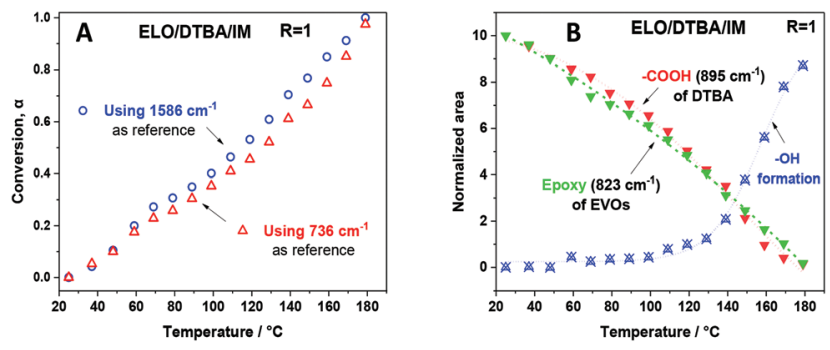

Fig. 5 (A) Conversion degree of epoxy groups calculated using two methods of calculation: (1) using peak at $1586 \mathrm{~cm}^{-1}$ as reference; (2) using peak at $736 \mathrm{~cm}^{-1}$ as reference; (B) Evolution of functional groups including epoxy, $-\mathrm{COOH},-\mathrm{OH}$, using the peak at $1586 \mathrm{~cm}^{-1}$ as reference, during curing of ELO/DTBA/IM mixture.

The first method uses the peak at $1586 \mathrm{~cm}^{-1}$ as the reference band. This band corresponds to $\mathrm{C}=\mathrm{C}$ stretching in the aromatic ring. The second method uses the peak at about $736 \mathrm{~cm}^{-1}$ as a reference, which is attributed to C-H bending of the ortho-substituted aromatic ring. The corresponding results are presented in Fig. 5A. The results obtained by both methods allowed confirmation of the reference peak choice and of the conversion calculation.

For the EPLO/IM mixture, a slight decrease in the band intensity of the epoxy rings at 847 and $823 \mathrm{~cm}^{-1}$ is observed. Moreover, we can remark that this band remains present in the case of ELO/IM mixture while the temperature increases up to $180{ }^{\circ} \mathrm{C}$. This indicates that some epoxy rings are still present, whereas some of them have already reacted with IM to form an epoxide/imidazole intermediary complex as shown in Scheme 1. During the heating of the DTBA/IM mixture we observe widening of the absorption bands at $1674 \mathrm{~cm}^{-1}$, $1463-1391 \mathrm{~cm}^{-1}$ and $\sim 895 \mathrm{~cm}^{-1}$. This suggests the formation of carboxylate groups $\left(\mathrm{COO}^{-}\right)$due to the basicity of IM.

Concerning the EVO/DTBA/IM reactive mixture, the FT-IR spectra recorded during the curing reveal the gradual diminution of the peaks at 1678 and $895 \mathrm{~cm}^{-1}$, corresponding to the $\mathrm{C}=\mathrm{O}$ stretching and $\mathrm{O}-\mathrm{H}$ bending of DTBA, together with the twin oxirane $\mathrm{C}-\mathrm{O}$ bands of $\mathrm{EVO}$ at 823 and $847 \mathrm{~cm}^{-1}$. At the same time, a progressive increase in the intensity of the peak at $3600 \mathrm{~cm}^{-1}$, characteristic of the hydroxyl groups $(-\mathrm{OH})$, and the appearance of a new ester peak located at about $1705 \mathrm{~cm}^{-1}$ are observed. The epoxy, carboxyl and ester groups absorb in 

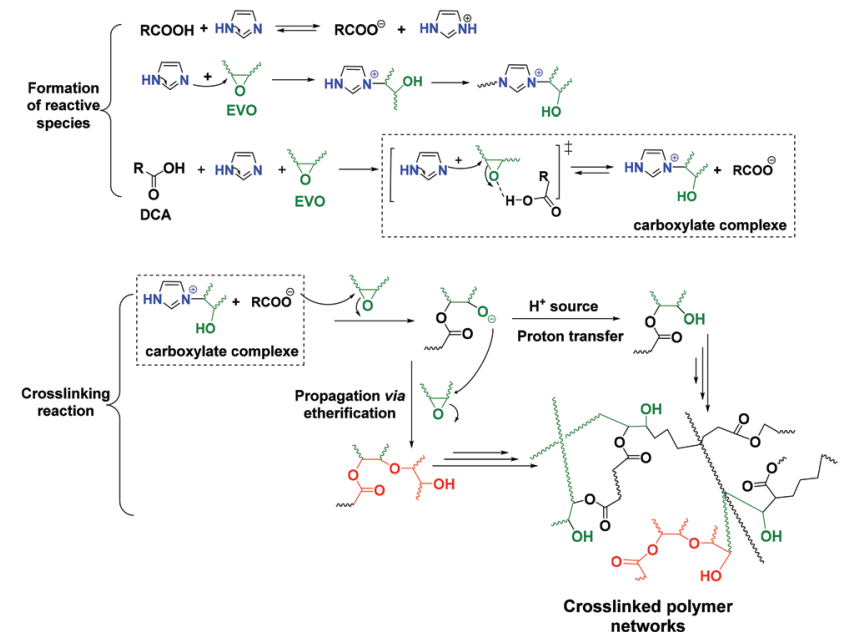

Scheme 1 Proposed reaction mechanism of the EVO/DCA system in the presence of imidazole as initiator.

the same region at $1315-1200 \mathrm{~cm}^{-1}$, related in each case to the stretching vibration of the $\mathrm{C}-\mathrm{O}$ bond. The decreases in absorption intensity are attributed to the consumption of epoxy and carboxyl groups while the increases in intensity are attributed to the formation of new ester groups. All these changes imply that the epoxy-acid crosslinking occurs via polyaddition esterification. It is proposed that IM initiates the reaction, acting by nucleophilic attack on the epoxy ring, which is activated by the DTBA H-donor. This process leads to the formation of imidazolium-carboxylate complexes via hydrogen bonding in the transition state (Scheme 1), selectively propagating the copolymerization. A proposed mechanism for the EVO/DCA reaction in the presence of imidazole as initiator is presented in Scheme 1.

When the epoxy/acid ratio $R=1$, the disappearance at the same rate of the $-\mathrm{COOH}$ absorption at $895 \mathrm{~cm}^{-1}$ and the epoxide absorption at about 823 and $847 \mathrm{~cm}^{-1}$, as shown in Fig. 5B, is concomitant with the appearance and increase in intensity of the $\mathrm{C}=\mathrm{O}_{\text {ester }}$ absorption at $1705 \mathrm{~cm}^{-1}$ and of the $-\mathrm{OH}$ absorption at $3650 \mathrm{~cm}^{-1}$. These structural changes are proof that epoxy-acid esterifications occur predominantly via a copolymerization reaction.

\section{Influence of heating rate on ELO/DTBA/IM curing}

FT-IR spectroscopy was employed to monitor the structural changes during the curing of the ELO/DTBA system $(R=1)$ at different heating rates, namely $5,10,15$, and $20{ }^{\circ} \mathrm{C} \min ^{-1}$. The corresponding results are presented in Fig. 6.

Fig. 6A indicates clearly that the heating rate had a great influence on the conversion degree. As expected, the curing reaction accelerates on increasing the heating rate. The relationship between reaction rate $(\mathrm{d} \alpha / \mathrm{d} t)$ and curing time is depicted in Fig. 6B. The reaction rate increases at higher heating rates during the entire conversion. Obviously, the fastest reaction rate was obtained with a heating rate of $20{ }^{\circ} \mathrm{C}$ $\min ^{-1}$, at which a strong reaction acceleration is observed. The
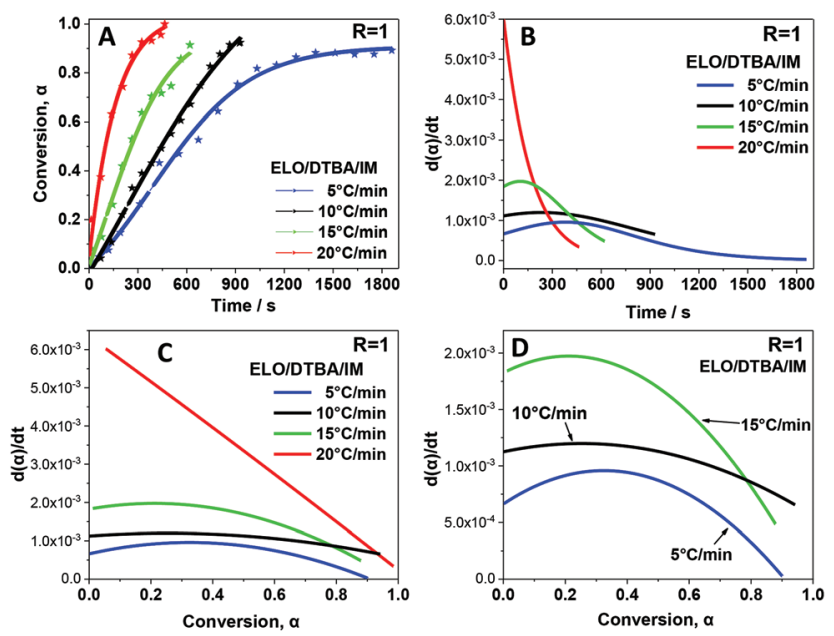

Fig. 6 (A) Plot of the degree of conversion $\alpha$ as a function of curing time at different heating rates; $(B)$ plot of reaction rate versus time; (C and $\mathrm{D})$ plot of reaction rate versus conversion degree.

maximum rate of reaction is achieved during the first minutes and then the rate decreases very fast. Moreover, this high heating rate is not preferred for polymer production since we cannot control the network formation as the rate of reaction is so high.

For the other three heating rates, 5, 10, and $15^{\circ} \mathrm{C} \min ^{-1}$, we can observe in Fig. 6 that the curing kinetics can be divided into two stages. The conversion rate rises slowly and gradually reaches a maximum value, then decreases progressively at the end. This suggests that the heating rate has a big impact on the curing kinetics and thus strongly affects the reaction mechanism in the first step of the non-isothermal curing process.

\section{Influence of EVO monomer reactivity}

The effect of the EVO's chemical structure on the EVO/DTBA/ IM curing was studied using stoichiometric amounts of epoxy/ acid compounds $(R=1)$. This ratio was revealed to be the most favorable for EVO/DCA curing, avoiding secondary reactions (etherification, Fisher esterification). Under this condition $(R=1)$, the copolymerization reaction is more selective, leading to the formation of hydroxyl ester products.

The plots of degree of conversion $(\alpha)$ as a function of the curing temperature and of time are exhibited in Fig. 7. The initial slope of the conversion curve is sharpest for EPLO followed by the ELO and ESFO systems. However, the slope gradually decreases after a certain temperature $\left(T \sim 110^{\circ} \mathrm{C}\right)$. This result is explained in the literature for epoxy/anhydride systems by the change from a kinetically-controlled stage to a diffusion-controlled stage. ${ }^{51,52,53}$ The authors interpret this result by an autocatalyzed-initiated mechanism for the curing process with the initial stage consisting of the formation of a "reactive" species (i.e. zwitterion-carboxylate). In the second stage, as the curing reaction proceeds, the reaction rate increases significantly to a maximum value when the concen- 

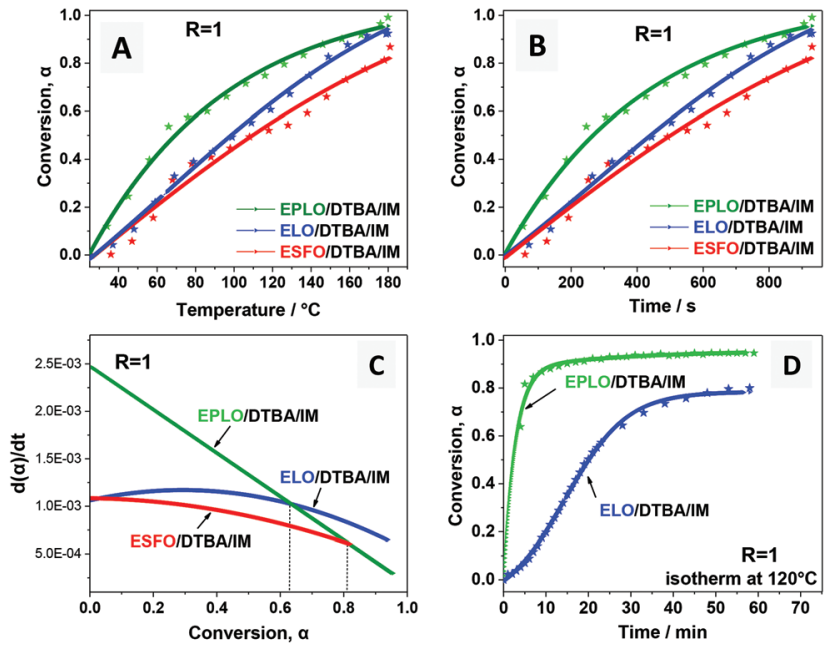

Fig. 7 Conversion $(\alpha)$ of EVO/DTBA/IM curing systems obtained by FT-IR analysis: (A) as a function of temperature; (B) as a function of time, (C) reaction rate as a function of conversion, (D) conversion as a function of time obtained by isothermal FT-IR at $120{ }^{\circ} \mathrm{C}$ for the EPLO and ELO/ DTBA/IM systems.

tration of the intermediate species is sufficiently high to promote and accelerate the autocatalytic reaction. ${ }^{53}$ Then, in the last stage, the reaction rate decreases due to the depletion of monomers, the inaccessibility of functional groups because of the steric hindrance, and the increased viscosity of the crosslinked media. The curing reaction is then predominantly controlled by the diffusion factor.

Fig. 7 exhibits the influence of epoxy content on the reactivity of the EVO/DTBA/IM systems. It can be clearly seen that as the epoxy content increases, the reaction starts earlier and the crosslinking rate is enhanced together with the conversion, in the order: ESFO < ELO < EPLO. This observation was confirmed by isothermal FT-IR analysis at $120^{\circ} \mathrm{C}$ (Fig. 7D) along with the apparent activation energy values ( $E_{\mathrm{a}}$, Table 1$)$. However, we observe also that at low conversion, at the very beginning of the reaction, $(\alpha \ll 0.1)$, the reaction rate $(\mathrm{d} \alpha / \mathrm{d} t)$ is slightly higher for the ESFO/DTBA/IM system compared to ELO/DTBA/IM, even though the epoxy content of ELO is higher (5.5 eq. per mole $v s .3 .7$ eq. per mole). This result can probably be related to the slightly lower molecular weight, and therefore higher accessibility of the oxirane groups during the first stage for ESFO (960 $\mathrm{g} \mathrm{mol}^{-1}$ vs. $980 \mathrm{~g} \mathrm{~mol}^{-1}$ for ELO). Moreover, the miscibility/compatibility between the monomer-hardener and reactive mixture-polymer product structures should also play an important role during the formation of the crosslinked network. Being the least viscous among the 3 EVOs, ESFO can provide better miscibility and interactions with the DTBA hardener, and consequently, a high reactivity early in the first stage. As also presented in Fig. 7C, the $\mathrm{d} \alpha / \mathrm{d} t=f$ (conversion) evolution is very different for the three EVOs. For example, when $\alpha>0.6$, the reaction rate is higher for ELO/DTBA/IM than for EPLO/DTBA/IM, which is probably due to the diffusion-controlled mechanism.

\section{Influence of the stoichiometry}

In this section, we study the effect of varying the epoxy/acid ratio on the crosslinking reactivity. The 3 EVOs were studied at 3 epoxy/acid ratios: $R=1, R=1.25$ and $R=2$. For instance, Fig. 8 exhibits the evolution of the FT-IR spectra of EPLO/ DTBA/IM mixtures with $R=1$ and $R=2$ during heating.

In addition, Fig. 9 shows the conversion profiles obtained from FT-IR studies during heating from 25 to $180{ }^{\circ} \mathrm{C}$ at $10^{\circ} \mathrm{C}$ $\mathrm{min}^{-1}$ for the $3 \mathrm{EVO} / \mathrm{DTBA} / \mathrm{IM}$ mixtures at ratios $R=1,1.25$ and 2. For all three EVOs, the common result is that in the first minutes, the reaction starts earlier when $R=1$. This result could be due to the presence of equivalent amounts of acidic protons and oxirane groups, favoring fast interaction during the first stage. Above certain temperatures, the conversion increases as the epoxy/acid ratio decreases. Moreover, the shape of the reaction rate curve changes when the $R$ ratio varies from 1 to 2 (Fig. 9B).

This result is probably due to the change in the reaction mechanism. It suggests that, when the epoxy concentration is in excess compared to the DTBA concentration, the polyaddition esterification reaction is predominant, due to the effect of imidazole on reaction selectivity, which is accompanied by a secondary etherification reaction. The formed alkoxide $\mathrm{R}-\mathrm{O}^{-}$can thus attack the oxirane groups present in a large amount in the mixture. Consequently, this could trigger propagation via etherification/epoxy ring-opening (Scheme 1). This observation confirms our previous hypothesis that the etherification competes with epoxy/acid copoly-

Table 1 Apparent activation energy $\left(E_{\mathrm{a}}\right)$ values obtained for EVO/DCA/IM $(R=1)$ systems using empirical fitting

\begin{tabular}{|c|c|c|c|c|c|c|c|c|}
\hline Kinetic model fit & & & DTBA & DPA & DTBA & DPA & DTBA & DPA \\
\hline \multirow{2}{*}{ First order } & For $\alpha \sim 0.4-0.8$ & $E_{2}\left(\mathrm{~kJ} \mathrm{~mol}^{-1}\right)$ & 8 & 14 & 11 & 21 & 25 & 9 \\
\hline & & $R^{2}$ & 0.9771 & 0.9618 & 0.9824 & 0.9352 & 0.914 & 0.9107 \\
\hline
\end{tabular}

$R^{2}$ : linear correlation coefficient. 
EPLO/DTBA/IM
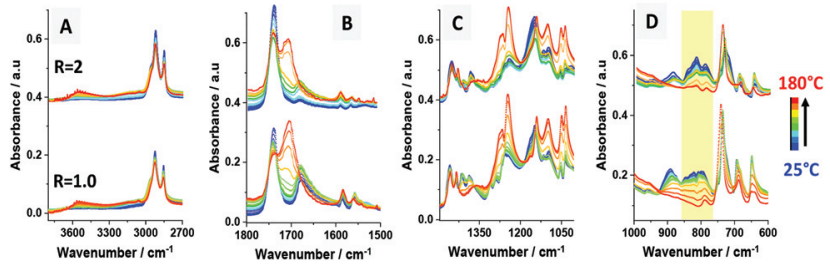

Fig. 8 Evolution of the FT-IR spectra of the EPLO/DTBA/IM mixtures with $R=1$ and $R=2$ during heating from 25 to $180^{\circ} \mathrm{C}$ at $10^{\circ} \mathrm{C} \mathrm{min}-1$ : (A) close-up of the region of $3800-2700 \mathrm{~cm}^{-1}$; (B) close-up of the region of $1800-1500 \mathrm{~cm}^{-1}$; (C) close-up of the region of $1500-1000 \mathrm{~cm}^{-1}$; (D) close-up of the region of $1000-600 \mathrm{~cm}^{-1}$.

merization in systems outside the stoichiometric ratio. In fact, when the concentration of DCA is higher than that of EVO, there is very little diffusion of reagents, due to the very high viscosity induced by the DCA, and consequently the conversion and reaction rate are lower compared with those of the $R=1$ system.

\section{Influence of the hardener structure}

The effect of the chemical structure of the aromatic diacid hardener was studied for $R=1$. A comparison is made here between DTBA and diphenic acid (DPA), an aromatic hardener which doesn't contain disulfide S-S bonds. Regarding the chemical structures of DPA and DTBA (Chart 1), it is obvious that the presence of sulphur in the $\beta$ position of the carboxylic group in the DTBA molecule increases the reactivity of its acidic proton toward the epoxy group. ${ }^{54}$ As expected, higher reactivity of DTBA towards oxirane groups is observed for all EVOs during the first steps (lower temperature). For example, in the case of the EPLO/DTBA/IM system the conversion $\alpha=$ 0.5 is achieved at a temperature of $\sim 70{ }^{\circ} \mathrm{C}$ whereas in the case of EPLO/DPA/IM the same conversion is achieved only at around $97^{\circ} \mathrm{C}\left(\Delta T \sim 27^{\circ} \mathrm{C}\right)$ (Fig. 10).

In contrast, DPA become more reactive at higher conversions: $\sim 0.88$ for EPLO/DPA/IM, and 0.60 and 0.38 respectively for ELO/DPA/IM and ESFO/DPA/IM. This could be due to the lower melting temperature of DPA $\left(230^{\circ} \mathrm{C} v s .290^{\circ} \mathrm{C}\right.$ for DTBA $)$
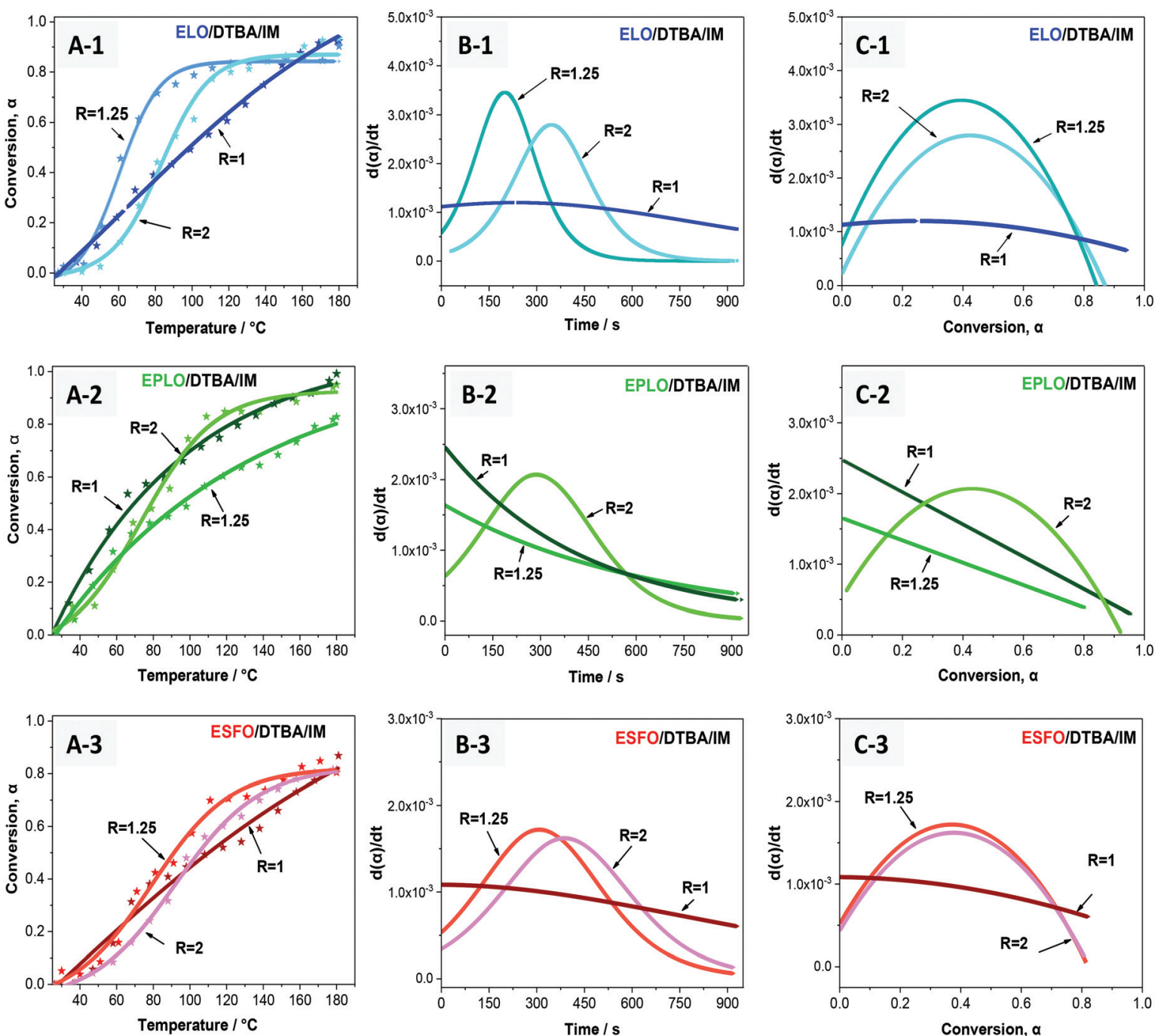

Fig. 9 Plots of conversion $(\alpha)$ as a function of temperature (A), and plots of reaction rate $\mathrm{d} \alpha / \mathrm{d} t$ as a function of time (B) and conversion $(\alpha)(\mathrm{C})$. 
Epoxidized Vegetable Oils (EVO)
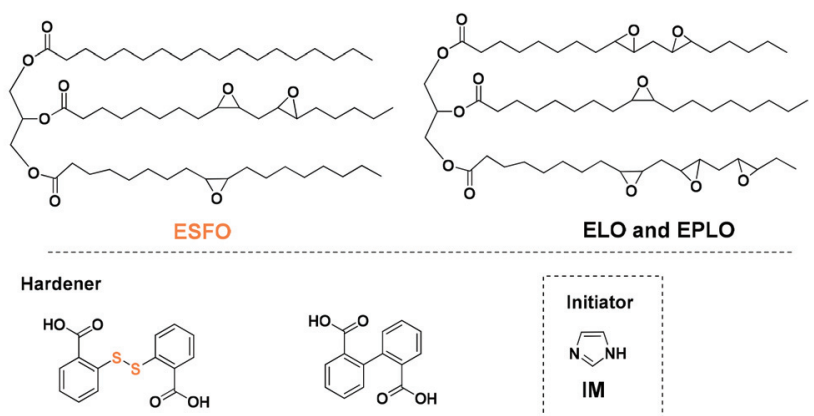

DTBA
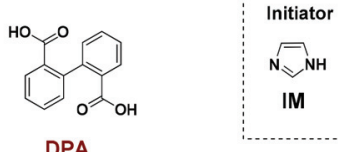

IM

Chart 1 Chemical structures of epoxidized safflower oil (ESFO), epoxidized linseed oil (ELO) and epoxidized perilla oil (EPLO), the two hardeners dithiodibenzoic acid (DTBA) and diphenic acid (DPA), and the imidazole (IM) initiator used in this study.


Fig. 10 Conversion $(\alpha)$ of EVO/DTBA/IM systems as a function of temperature in comparison with EVO/DPA/IM, obtained by FT-IR analysis: EPLO/DTBA/IM vs. EPLO/DPA/IM (A); ELO/DTBA/IM vs. ELO/DPA/IM (B) and ESFO/DTBA/IM vs. ESFO/DPA/IM (C).

and therefore the lower viscosity of the mixtures. In the first steps, the epoxy-acid reaction is controlled by the reactivity of the comonomers. Thereafter, the crosslinking becomes diffusion-controlled.

Obviously, the epoxy/acid ratio affects both the reaction order and the $E_{\mathrm{a}}$ of the crosslinking reaction. The amount of catalyst or initiator affects only the apparent $E_{\mathrm{a}}$. For the same epoxy/acid ratio, the reaction order $(n)$ should remain unaffected. Consequently, we applied $n=1$ (first order) and $n$ $=2$ (second order) to estimate the $E_{\mathrm{a}}$ for the 3 EVOs reacting with the two aromatic dicarboxylic acids at $R=1$ in order to compare the reactivity of these systems. The same methods are applied for all crosslinking systems and the fitted results are given in Table 1.
For both applied methods, the same trend in activation energy was obtained: higher $E_{\mathrm{a}}$ values for ESFO among the 3 EVOs and for DTBA among the two hardeners. The $E_{\mathrm{a}}$ is higher during the first stages of the curing process, i.e. for $\alpha<$ 0.4 and then it diminishes when $\alpha$ increases. This can be explained by a combination of physical and chemical factors that act during the curing process. It is supposed that at the beginning of the curing, the reaction mechanism is dominated by the chemical reactivity and in the later stages it is controlled by physical diffusion. The $E_{\mathrm{a}}$ values are in the range of $40-100 \mathrm{~kJ} \mathrm{~mol}^{-1}$ and decrease from 74 to $48 \mathrm{~kJ} \mathrm{~mol}^{-1}$ when the epoxy content increases from 3.7 eq. per mole to 8.0 eq. per mole in the case of the EVO/DTBA/IM systems. The same relationship between $E_{\mathrm{a}}$ and epoxy content is observed for the

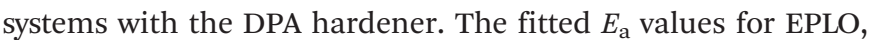
ELO, and ESFO are 43, 53, and $66 \mathrm{~kJ} \mathrm{~mol}^{-1}$, respectively, assuming that the reaction mechanism is first order.

\section{Kinetic analysis}

Generally, epoxy/hardener crosslinking is characterized by a quite complex mechanism. The competing reactions involved are related to different kinetic equations, but the rate of reaction can be formulated from three different variables, namely temperature $T$, extent of conversion $\alpha$, and pressure $P$, as presented in eqn (1):

$$
\frac{\mathrm{d} \alpha}{\mathrm{d} t}=k(T) f(\alpha) h(P)
$$

In most curing processes, the effect of pressure is usually not considered because no gas takes part in the reactions, so the rate of reaction depends on only two variables $(T, \alpha)$

$$
\frac{\mathrm{d} \alpha}{\mathrm{d} t}=k(T) f(\alpha)
$$

where $k(T)$ is the temperature-dependent reaction rate constant and is defined by the Arrhenius equation:

$$
k(T)=A \exp \left(\frac{-E a}{R T}\right)(\alpha)
$$

where $E_{\text {a }}$ represents the activation energy, $R$ is the universal gas constant equal to $8.3145 \mathrm{~J} \mathrm{~K}^{-1}$ and $A$ is the pre-exponential factor. The function $f(\alpha)$ is the model representing the reaction mechanism governing the curing process, dependent on the curing degree. It can be simulated by various reaction models. In general, $f(\alpha)$ for epoxy curing is usually hypothesized to follow an $n^{\text {th }}$ order reaction model.

After some derivation, the following equation is obtained:

$$
\ln \frac{(G(\alpha))}{T^{2}}=\ln (A)-\frac{E_{\mathrm{a}}}{R T}
$$

By plotting $\ln \frac{(G(\alpha))}{T^{2}}$ versus $1 / T$, a linear dependence is obtained with a slope of $-E_{\mathrm{a}} / R$. Therefore, the activation energy $\left(E_{\mathrm{a}}\right)$ can be calculated from this slope. The reaction model equations are shown in Table 2. The $f(\alpha)$ model fitting is validated by the best correlation coefficient $\left(R^{2}\right)$ for the 
Table 2 Possible expressions for $f(\alpha)$ and $G(\alpha)$ for different kinetic models

\begin{tabular}{lcl}
\hline $\begin{array}{l}\text { Third order } \\
n^{\text {th }} \text { order }\end{array}$ & $(1-\alpha)^{3}$ & {$\left[(1-\alpha)^{-2}-1\right] / 2$} \\
Limiting surface reaction between both phases & {$\left[(1-\alpha)^{1-n}-1\right] /(n-1)$} \\
One dimension & 1 & $\alpha$ \\
Two dimensions & $2(1-\alpha)^{1 / 2}$ & $1-(1-\alpha)^{1 / 2}$ \\
Three dimensions & $3(1-\alpha)^{2 / 3}$ & $1-(1-\alpha)^{1 / 3}$ \\
Diffusion & & \\
One-way transport & $1 / 2 \alpha$ & $\alpha^{2}$ \\
Two-way transport & {$[-\ln (1-\alpha)]^{-1}$} & $\alpha+(1-\alpha) \ln (1-\alpha)$
\end{tabular}

straight line $\left(\ln \frac{(G(\alpha))}{T^{2}}\right.$ versus $\left.1 / T\right)$ as obtained by empirical methods. In this study, when $R=1$, we assume that only epoxy/acid esterifications occur during the curing. First order and second order kinetic analyses were applied to the FT-IR data.

\section{Conclusions}

This work explores the influence of EVO epoxy content on the kinetics of EVO/DCA copolymerization. The obtained results reveal that the EVO/DCA reaction depends strongly not only on the degree of epoxidation but also on the type of vegetable oil (i.e. degree of unsaturation).

Moreover, in this work, for the first time the activation energy was calculated via only the simple FT-IR technique. We have successfully demonstrated that a structure-reactivity relationship exists in these systems. Thus, EPLO is revealed as the most reactive monomer as a consequence of its high epoxy content. The obtained results prove that it is possible to predict and modulate the EVO/DCA reaction kinetics in order to design bio-based thermosets with desirable and competitive properties. The influence of diacid chemical structure was also investigated. The presence of dynamic S-S bonding in the $\beta$ position of the carboxylic group increases the reactivity of the acidic proton toward the epoxy group, and therefore produces an increase in reactivity.

\section{Experimental section}

\section{Materials}

Epoxidized linseed oil (ELO, average molecular weight $=980$ $\mathrm{Da}$; average functionality $=5.5$ epoxides per triglyceride) was supplied by Valtris Specialty Chemicals. Epoxidized perilla oil (EPLO, average molecular weight $=1227 \mathrm{Da}$; average functionality $=8.0$ epoxides per triglyceride) and epoxidized safflower oil (ESFO, average molecular weight $=960 \mathrm{Da}$; average functionality $=3.7$ epoxides per triglyceride) were supplied by Specific Polymers. 2,2'-Dithiodibenzoic acid (DTBA, $\geq 95 \%$ ), diphenic acid (DPA, $\geq 97 \%$ ), and imidazole (IM, $\geq 99 \%$ ) were purchased from Sigma Aldrich. All chemical compounds were used as received without any further purification and are presented in Chart 1.

\section{Fourier-transform infrared spectroscopy (FT-IR)}

The crosslinking reaction kinetics was monitored in situ using a Thermo Scientific Nicolet iS50 FT-IR spectrometer with a deuterated L-alanine doped triglycine sulfate (DLaTGS) detector in attenuated total reflectance (ATR) mode. To prepare the formulations, all components were pre-mixed at room temperature, and then cured in situ during heating from 25 to $180{ }^{\circ} \mathrm{C}$ at a selected heating rate $\left(5,10,15\right.$ or $\left.20^{\circ} \mathrm{C} \mathrm{min}^{-1}\right)$. The FT-IR spectra were recorded each minute during heating. The absorption bands were recorded in the range of $600-4000 \mathrm{~cm}^{-1}$ with 32 scans and a resolution of $2 \mathrm{~cm}^{-1}$. The data were analysed using the OMNIC software. The conversion of functional groups at time $t$ is denoted by $\alpha$ and defined by eqn (5).

$$
\alpha=\frac{\left(\frac{A_{\text {functional groups }}}{A_{\text {ref }}}\right) 0-\left(\frac{A_{\text {functional groups }}}{A_{\text {ref }}}\right) t}{\left(\frac{A_{\text {functional groups }}}{A_{\text {ref }}}\right) 0}
$$

The area of the absorbance peaks was calculated and reported for different times $\left(A_{t}\right)$ after the initial time $\left(A_{0}\right)$. The band at about $823 \mathrm{~cm}^{-1}$ corresponds to the oxirane $\mathrm{C}-\mathrm{O}$ groups and that at about $895 \mathrm{~cm}^{-1}$ corresponds to the carboxylic groups. Two methods of calculation were applied for the epoxy conversion. The first method used the peak at $1586 \mathrm{~cm}^{-1}$ as the reference band, which corresponds to $\delta_{\mathrm{C}-\mathrm{C}}$ for the aromatic rings. The second method used the peak at about $736 \mathrm{~cm}^{-1}$ as a reference, which is attributed to $\mathrm{C}-\mathrm{H}$ bending of the ortho-substituted aromatic rings. Each sample was prepared and analysed in triplicate to ensure efficiency, reliability and reproducibility.

\section{Sample preparation}

The EVO/DCA copolymerizations were carried out in the presence of $1 \%(\mathrm{w} / \mathrm{w})$ of imidazole as initiator. The ratio $R$ is defined as the ratio of epoxy groups to carboxylic acid groups according to the literature. ${ }^{55}$ The initiator was firstly dissolved in EVO at around $80^{\circ} \mathrm{C}$ and the solution was mixed together. Then, at room temperature, the selected DCA was added and the solution was mixed until complete homogenization. The freshly prepared samples were analysed by FT-IR to follow the copolymerization reaction.

\section{Abbreviations}

EVO Epoxidized vegetable oil

ELO Epoxidized linseed oil

EPLO Epoxidized perilla oil

ESFO Epoxidized safflower oil

DCA Dicarboxylic acid

DTBA 2,2'-Dithiodibenzoic acid

DPA Diphenic acid

IM Imidazole

FT-IR Fourier Transform Infrared Spectroscopy

vs. versus 


\section{Conflicts of interest}

There are no conflicts to declare.

\section{Acknowledgements}

This work was supported by the ECOXY project funded by the Bio Based Industries Joint Undertaking under European Union Horizon 2020 Research and Innovation Program (Grant agreement no. 744311).

\section{Notes and references}

1 G. Mashouf Roudsari, A. K. Mohanty and M. Misra, Green Approaches To Engineer Tough Biobased Epoxies: A Review, ACS Sustainable Chem. Eng., 2017, 5(11), 9528-9541.

2 E. Ramon, C. Sguazzo and P. M. G. P. Moreira, A Review of Recent Research on Bio-Based Epoxy Systems for Engineering Applications and Potentialities in the Aviation Sector, Aerospace, 2018, 5(4), 110.

3 S. Kumar, S. K. Samal, S. Mohanty and S. K. Nayak, Recent Development of Biobased Epoxy Resins: A Review, Polym.Plast. Technol. Eng., 2018, 57(3), 133-155.

4 Y. Rok Ham, S. Hee Kim, Y. Shin, D. Lee, M. Yang, J. Hye Min and J. Shin, A comparison of some imidazoles in the curing of epoxy resin, J. Ind. Eng. Chem., 2010, 16, 556-559.

5 J. M. Pin, N. Guigo, L. Vincent, N. Sbirrazzuoli and A. Mija, Copolymerization as a Strategy to Combine Epoxidized Linseed Oil and Furfuryl Alcohol: The Design of a Fully Bio-Based Thermoset, ChemSusChem, 2015, 8(24), 40984098.

6 A.-S. Mora, R. Tayouo, B. Boutevin, G. David and S. Caillol, Vanillin-derived amines for bio-based thermosets, Green Chem., 2018, 20(17), 4075-4084.

7 S. Dhers, G. Vantomme and L. Avérous, A fully bio-based polyimine vitrimer derived from fructose, Green Chem., 2019, 21(7), 1596-1601.

8 M. Shibata and T. Ohkita, Fully biobased epoxy resin systems composed of a vanillin-derived epoxy resin and renewable phenolic hardeners, Eur. Polym. J., 2017, 92, 165-173.

9 A. D. La Rosa, I. Blanco, D. R. Banatao, S. J. Pastine, A. Björklund and G. Cicala, Innovative chemical process for recycling thermosets cured with recyclamines by converting Bio-Epoxy Composites in Reusable Thermoplastic-An LCA Study, Materials, 2018, 11(3), 353.

10 J. C. Ronda, G. Lligadas, M. Galià and V. Cádiz, Vegetable oils as platform chemicals for polymer synthesis, Eur. J. Lipid Sci. Technol., 2011, 113(1), 46-58.

11 M. Stemmelen, V. Lapinte, J.-P. Habas and J.-J. Robin, Plant oil-based epoxy resins from fatty diamines and epoxidized vegetable oil, Eur. Polym. J., 2015, 68, 536-545.

12 C. Ding, P. S. Shuttleworth, S. Makin, J. H. Clark and A. S. Matharu, New insights into the curing of epoxidized linseed oil with dicarboxylic acids, Green Chem., 2015, 17(7), 4000-4008.

13 S. G. Tan and W. S. Chow, Curing Characteristics and Thermal Properties of Epoxidized Soybean Oil Based Thermosetting Resin, J. Am. Oil Chem. Soc., 2011, 88(7), 915-923.

14 F. I. Altuna, V. Pettarin and R. J. J. Williams, Self-healable polymer networks based on the cross-linking of epoxidised soybean oil by an aqueous citric acid solution, Green Chem., 2013, 15(12), 3360-3366.

15 C. Vilela, A. F. Sousa, A. C. Fonseca, A. C. Serra, J. F. J. Coelho, C. S. R. Freire and A. J. D. Silvestre, The quest for sustainable polyesters - insights into the future, Polym. Chem., 2014, 5(9), 3119-3141.

16 M. A. R. Meier, J. O. Metzger and U. S. Schubert, Plant oil renewable resources as green alternatives in polymer science, Chem. Soc. Rev., 2007, 36(11), 1788-1802.

17 L. Montero de Espinosa and M. A. R. Meier, Plant oils: The perfect renewable resource for polymer science?!, Eur. Polym. J., 2011, 47(5), 837-852.

18 Y. Chen, Z. Xi and L. Zhao, Curing kinetics of bio-based epoxy resin based on epoxidized soybean oil and green curing agent, AIChE J., 2017, 63(1), 147-153.

19 J. D. Espinoza-Perez, B. A. Nerenz, D. M. Haagenson, Z. Chen, C. A. Ulven and D. P. Wiesenborn, Comparison of curing agents for epoxidized vegetable oils applied to composites, Polym. Compos., 2011, 32(11), 1806-1816.

20 X. Huang, X. Yang, H. Liu, S. Shang, Z. Cai and K. Wu, Biobased thermosetting epoxy foams from epoxidized soybean oil and rosin with enhanced properties, Ind. Crops Prod., 2019, 139, 111540.

21 K. Thiele, N. Eversmann, A. Krombholz and D. PufkyHeinrich, Bio-Based Epoxy Resins Based on Linseed Oil Cured with Naturally Occurring Acids, Polymers, 2019, 11(9), 1409.

22 G. S. Sudha, H. Kalita, S. Mohanty and S. K. Nayak, Biobased epoxy blends from epoxidized castor oil: Effect on mechanical, thermal, and morphological properties, Macromol. Res., 2017, 25(5), 420-430.

23 C. Zhao, G. Zhang and L. Zhao, Effect of curing agent and temperature on the rheological behavior of epoxy resin systems, Molecules, 2012, 17(7), 8587-8594.

24 I. Blanco, L. Oliveri, G. Cicala and A. Recca, Effects of novel reactive toughening agent on thermal stability of epoxy resin, J. Therm. Anal. Calorim., 2012, 108(2), 685-693.

25 T. Boonlert-uthai, C. Samthong and A. Somwangthanaroj, Synthesis, thermal properties and curing kinetics of hyperbranched BPA/PEG epoxy resin, Polymers, 2019, 11(10), 1545.

26 H. Q. Pham and M. J. Marks, Epoxy Resins, in Ullmann's Encyclopedia of Industrial Chemistry, 2000.

27 D. Montarnal, M. Capelot, F. Tournilhac and L. Leibler, Silica-Like Malleable Materials from Permanent Organic Networks, Science, 2011, 334(6058), 965.

28 D. Montarnal, F. Tournilhac, M. Hidalgo and L. Leibler, Epoxy-based networks combining chemical and supramole- 
cular hydrogen-bonding crosslinks, J. Polym. Sci., Part A: Polym. Chem., 2010, 48(5), 1133-1141.

29 K. Yu, P. Taynton, W. Zhang, M. L. Dunn and H. J. Qi, Reprocessing and recycling of thermosetting polymers based on bond exchange reactions, $R S C A d v$., 2014, 4(20), 10108-10117.

30 M. M. Obadia, B. P. Mudraboyina, A. Serghei, D. Montarnal and E. Drockenmuller, Reprocessing and Recycling of Highly Cross-Linked Ion-Conducting Networks through Transalkylation Exchanges of C-N Bonds, J. Am. Chem. Soc., 2015, 137(18), 6078-6083.

31 Y.-X. Lu, F. Tournilhac, L. Leibler and Z. Guan, Making Insoluble Polymer Networks Malleable via Olefin Metathesis, J. Am. Chem. Soc., 2012, 134(20), 8424-8427.

32 A. Ruiz de Luzuriaga, R. Martin, N. Markaide, A. Rekondo, G. Cabañero, J. Rodríguez and I. Odriozola, Epoxy resin with exchangeable disulfide crosslinks to obtain reprocessable, repairable and recyclable fiber-reinforced thermoset composites, Mater. Horiz., 2016, 3(3), 241-247.

33 Z. Ma, Y. Wang, J. Zhu, J. Yu and Z. Hu, Bio-based epoxy vitrimers: Reprocessibility, controllable shape memory, and degradability, J. Polym. Sci., Part A: Polym. Chem., 2017, 55(10), 1790-1799.

34 T. Ohishi, Y. Iki, K. Imato, Y. Higaki, A. Takahara and H. Otsuka, Insertion Metathesis Depolymerization of Aromatic Disulfide-containing Dynamic Covalent Polymers under Weak Intensity Photoirradiation, Chem. Lett., 2013, 42(11), 1346-1348.

35 Y. Amamoto, J. Kamada, H. Otsuka, A. Takahara and K. Matyjaszewski, Repeatable Photoinduced Self-Healing of Covalently Cross-Linked Polymers through Reshuffling of Trithiocarbonate Units, Angew. Chem., Int. Ed., 2011, 50(7), 1660-1663.

36 S. Burattini, B. W. Greenland, D. H. Merino, W. Weng, J. Seppala, H. M. Colquhoun, W. Hayes, M. E. Mackay, I. W. Hamley and S. J. Rowan, A Healable Supramolecular Polymer Blend Based on Aromatic $\pi-\pi$ Stacking and Hydrogen-Bonding Interactions, J. Am. Chem. Soc., 2010, 132(34), 12051-12058.

37 L. Cao, J. Fan, J. Huang and Y. Chen, A robust and stretchable cross-linked rubber network with recyclable and selfhealable capabilities based on dynamic covalent bonds, J. Mater. Chem. A, 2019, 7(9), 4922-4933.

38 Z. Q. Lei, H. P. Xiang, Y. J. Yuan, M. Z. Rong and M. Q. Zhang, Room-Temperature Self-Healable and Remoldable Cross-linked Polymer Based on the Dynamic Exchange of Disulfide Bonds, Chem. Mater., 2014, 26(6), 2038-2046.

39 M. Chen, L. Zhou, Y. Wu, X. Zhao and Y. Zhang, Rapid Stress Relaxation and Moderate Temperature of Malleability Enabled by the Synergy of Disulfide Metathesis and Carboxylate Transesterification in Epoxy Vitrimers, ACS Macro Lett., 2019, 8(3), 255-260.

40 J. Canadell, H. Goossens and B. Klumperman, Self-Healing Materials Based on Disulfide Links, Macromolecules, 2011, 44(8), 2536-2541.
41 N. Boquillon and C. Fringant, Polymer networks derived from curing of epoxidised linseed oil: influence of different catalysts and anhydride hardeners, Polymer, 2000, 41(24), 8603-8613.

42 C. Li, J. Sung and X. S. Sun, Network from Dihydrocoumarin via Solvent-Free Metal-Mediated Pathway: A Potential Structure for Substantial Toughness Improvement of Epoxidized Plant Oil Materials, ACS Sustainable Chem. Eng., 2016, 4(3), 1231-1239.

43 B. K. Ahn, S. Kraft, D. Wang and X. S. Sun, Thermally Stable, Transparent, Pressure-Sensitive Adhesives from Epoxidized and Dihydroxyl Soybean Oil, Biomacromolecules, 2011, 12(5), 1839-1843.

44 J. M. España, L. Sánchez-Nacher, T. Boronat, V. Fombuena and R. Balart, Properties of Biobased Epoxy Resins from Epoxidized Soybean Oil (ESBO) Cured with Maleic Anhydride (MA), J. Am. Oil Chem. Soc., 2012, 89(11), 20672075.

45 T.-N. Tran, C. Di Mauro, A. Graillot and A. Mija, Chemical Reactivity and the Influence of Initiators on the Epoxidized Vegetable Oil/Dicarboxylic Acid System, Macromolecules, 2020, 53(7), 2526-2538.

46 J.-M. Pin, N. Guigo, L. Vincent, N. Sbirrazzuoli and A. Mija, Copolymerization as a Strategy to Combine Epoxidized Linseed Oil and Furfuryl Alcohol: The Design of a Fully Bio-Based Thermoset, ChemSusChem, 2015, 8(24), 41494161.

47 A. R. Mahendran, N. Aust, G. Wuzella and A. Kandelbauer, Synthesis and Characterization of a Bio-Based Resin from Linseed Oil, Macromol. Symp., 2012, 311(1), 18-27.

48 G. Falco, N. Sbirrazzuoli and A. Mija, Biomass derived epoxy systems: From reactivity to final properties, Mater. Today Commun., 2019, 21, 100683.

49 J.-J. Max and C. Chapados, Infrared Spectroscopy of Aqueous Carboxylic Acids: Comparison between Different Acids and Their Salts, J. Phys. Chem. A, 2004, 108(16), 33243337.

50 S. G. Tan and W. S. Chow, Thermal properties of anhydride-cured bio-based epoxy blends, J. Therm. Anal. Calorim., 2010, 101, 1051-1058.

$51 \mathrm{~J}$. Gao and Y. Li, Curing kinetics and thermal property characterization of a bisphenol-S epoxy resin and DDS system, Polym. Int., 2000, 49(12), 1590-1595.

52 S. G. Tan, Z. Ahmad and W. S. Chow, Relationships of cure kinetics and processing for epoxidized soybean oil bio-thermoset, Ind. Crops Prod., 2013, 43, 378-385.

53 H. Wang, B. Liu, X. Liu, J. Zhang and M. Xian, Synthesis of biobased epoxy and curing agents using rosin and the study of cure reactions, Green Chem., 2008, 10(11), 11901196.

54 F. Jaillet, M. Desroches, R. Auvergne, B. Boutevin and S. Caillol, New biobased carboxylic acid hardeners for epoxy resins, Eur. J. Lipid Sci. Technol., 2013, 115(6), 698-708.

55 L. Matejka, S. Pokomy and K. Dusek, Network Formation Involving Epoxide and Carboxyl Groups, Pol. Bull., 1982, 7, 123-128. 